\title{
A BOUNDARY-LAYER PROBLEM IN FRESH-SALT GROUNDWATER FLOW
}

\author{
By C. J. VAN DUIJN \\ (Department of Mathematics, Delft University of Technology, Julianalaan 132, \\ 2628 BL Delft, The Netherlands) \\ and L. A. PELETIER \\ (Mathematical Institute, Leiden University, The Netherlands)
}

[Received 7 June 1990. Revise 10 March 1991]

\section{S UM M A R Y}

In this paper we investigate the boundary layer which arises between the parallel flow of fresh and salt ground water as occurs in vertical aquifers. It is caused by small molecular diffusion and transversal dispersion brought about by the porous environment. A self-similar transformation leads to a variant of the Falkner-Skan equation. Existence, uniqueness and various properties of the solution, such as monotonicity and asymptotic behaviour, are established and the influence of the ratio of molecular diffusion to transversal dispersion is analysed. Some numerical results are presented.

\section{Introduction}

CONSIDER the two-dimensional flow of an incompressible fluid through a homogeneous and isotropic porous medium. The fluid has a constant dynamic viscosity $\mu>0$ and a variable density $\rho$, and the porous medium is characterized by a constant porosity $\Phi \in(0,1)$ and a constant permeability $\kappa>0$.

A typical example of such a situation arises in the flow of fresh and salt groundwater in a two-dimensional vertical aquifer $(\mathbf{1}, \mathbf{2}, \mathbf{3})$. In this application one has for the densities $\rho_{f}$ and $\rho_{s}$ of the fresh and of the salt groundwater, respectively:

$$
0<\rho_{f}<\rho<\rho_{s}<\infty
$$

throughout the entire flow domain. Assuming that the volume of the fluid is not affected by its density, the equations describing the problem result from fluid incompressibility:

$$
\operatorname{div} \bar{q}=0
$$

momentum balance (Darcy's law):

$$
\frac{\mu}{\kappa} \bar{q}+\operatorname{grad} p-\rho \bar{g}=0,
$$


and mass balance:

$$
\Phi \frac{\partial \rho}{\partial t}+\operatorname{div} \bar{F}=0
$$

In these equations, $\bar{q}=\left(q_{1}, q_{2}\right)=(u, v)$ denotes the specific discharge of the fluid, $p$ the fluid pressure and $\bar{g}$ the acceleration of gravity. In equation (1.4), the vector $\bar{F}$ denotes the mass flux. It is given by

$$
\bar{F}=\rho \bar{g}-D \operatorname{grad} \rho,
$$

where $D=\left(D_{i j}\right)$ is the hydrodynamic dispersion matrix defined by $(1, \mathrm{p} .612)$

$$
D_{\imath \jmath}=\left\{\alpha_{T}|\bar{q}|+\Phi D_{\mathrm{mol}}\right\} \delta_{i j}+\left(\alpha_{L}-\alpha_{T}\right) \frac{q_{t} q_{j}}{|\bar{q}|}
$$

Here $\alpha_{T}$ and $\alpha_{L}$ are positive constants with $\alpha_{T}<\alpha_{L}$. They are called the transversal and the longitudinal dispersion length and they describe the diffusion due to the mechanical dispersion caused by the randomness of the structure of the porous matrix. Further, $D_{\text {mol }}$ is a positive constant representing the molecular diffusion in the fluid. Finally $\delta_{i j}$ denotes the Kronecker delta and $|\cdot|$ the Euclidean norm in $\mathbb{R}^{2}$.

In this paper we study a particular flow problem, which was recently investigated by Uffink $(4,5)$. In Fig. 1 below we sketch the situation and explain some notation. In the region $\{x<0, z>0\}$ we suppose that fresh water $\left(\rho=\rho_{f}\right)$ has a uniform specific discharge $u=U, v=0$ along an impervious boundary $\{x<0, z=0\}$. In the region $\{x>0\}$ we suppose that fresh water is present far above the plane $\{z=0\}$ and salt water far below.

In the absence of diffusion or dispersion ( $D=0$ in (1.5)), an abrupt transition or interface exists between the fresh and the salt water. It is well known that this leads to a jump in the tangential component of the specific discharge along

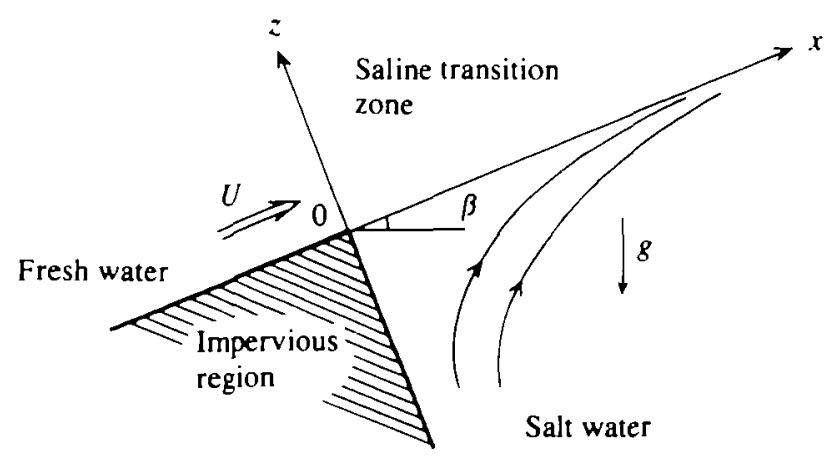

FIG. 1. Geometry of the problem 
the interface, the shear flow $(\mathbf{1}, \mathbf{6})$, of magnitude

$$
\left(\bar{q}_{f}-\bar{q}_{s}\right) \cdot \bar{t}=\left(\rho_{s}-\rho_{f}\right) g \frac{\kappa}{\mu} \sin \beta .
$$

Here $\bar{q}_{f}$ and $\bar{q}_{s}$ denote the specific discharge just above and below the interface and $\bar{t}$ is the tangential unit vector along the interface pointing in the direction of positive $x$. Thus, if we look for a flow configuration in which the salt water is stagnant, we must require that

$$
U=\left(\rho_{s}-\rho_{f}\right) g \frac{\kappa}{\mu} \sin \beta .
$$

This leads to a fresh-salt interface which coincides with the half-plane $\{x>0$, $z>0\}$.

In general, when dispersion is present, a transition zone will develop which will be thin if the coefficients $\alpha_{T}, \alpha_{L}$ and $D_{\text {mol }}$ in the dispersion matrix $D$ are small. In (4) Uffink presents a boundary-layer model to describe the steady mixing of the two fluids in this transition zone. Below we give the main steps of the derivation.

To eliminate the pressure from Darcy's law (1.3) we take the curl. This yields

$$
\operatorname{curl}\left(\frac{\mu}{\kappa} \bar{q}-\rho \bar{g}\right)=0
$$

or, written out in detail,

$$
\frac{\partial u}{\partial z}-\frac{\partial v}{\partial x}+\frac{\kappa}{\mu} g\left(\frac{\partial \rho}{\partial z} \sin \beta-\frac{\partial \rho}{\partial x} \cos \beta\right)=0
$$

The system of equations consisting of (1.2), (1.4) to (1.6) and (1.9) describes the mixing of the fluids.

To investigate the development of the transition zone, a singular perturbation method is used (7). Thus we get

$$
\delta^{2}=\alpha_{T} / L
$$

where $L$ is a characteristic length of the problem such as the distance to the origin. Introducing dimensionless variables

$$
x^{\prime}=x / L, \quad z^{\prime}=z /(L \delta), \quad u^{\prime}=u / U, \quad v^{\prime}=v /(U \delta)
$$

and

$$
\rho^{\prime}=\frac{\rho-\rho_{f}}{\rho_{s}-\rho_{f}}
$$


and omitting the primes again for convenience, (1.9) yields

$$
\frac{\partial u}{\partial z}-\delta^{2} \frac{\partial v}{\partial x}+\Gamma\left(\frac{\partial \rho}{\partial z} \sin \beta-\delta \frac{\partial \rho}{\partial x} \cos \beta\right)=0,
$$

where

$$
\Gamma=\frac{\rho_{s}-\rho_{f}}{U} g \frac{\kappa}{\mu} .
$$

If we now let $\delta \rightarrow 0$ and use (1.8), we obtain

$$
\frac{\partial u}{\partial z}+\frac{\partial \rho}{\partial z}=0 .
$$

Choosing $u=0$ deep in the salt-water region, where $\rho=1$, we obtain upon integration

$$
u+\rho=1 .
$$

This yields $u=1$ (or $u=U$ in the original variables) in the fresh-water region, far from the transition layer.

Similarly, we substitute (1.10) into equations (1.2) and (1.4) to (1.6) and let $\delta \rightarrow 0$. We then find the following set of boundary-layer equations for steady flow:

$$
\begin{aligned}
& \frac{\partial u}{\partial x}+\frac{\partial v}{\partial z}=0 \\
& u \frac{\partial \rho}{\partial x}+v \frac{\partial \rho}{\partial z}=\frac{\partial}{\partial z}\left\{(|u|+\lambda) \frac{\partial \rho}{\partial z}\right\} \\
& u=1-\rho .
\end{aligned}
$$

Here

$$
\lambda=\frac{\Phi D_{\text {mol }}}{\alpha_{T} U}
$$

denotes the ratio between molecular diffusion and transversal mechanical dispersion. The value of $\lambda$ depends on the type of soil: for homogeneous fine grained soils it can be large but for heterogeneous soils it is very small. Note that, using $(1.12)_{3}$ to eliminate $\rho,(1.12)_{2}$ can be written as

$$
u \frac{\partial u}{\partial x}+v \frac{\partial u}{\partial z}=\frac{\partial}{\partial z}\left\{(|u|+\lambda) \frac{\partial u}{\partial z}\right\}
$$

At this point it is worthwhile to compare equations (1.12) with the Prandtl boundary-layer equations (7). The additional term in $(1.12)_{4}$ is the degenerate diffusion term $\left(|u| u_{z}\right)_{r}$, where the subscript $z$ denotes differentiation with respect 
to $z$. The appearance of this term enables us to consider solutions in the limit as $\lambda \rightarrow 0$.

We consider (1.12) in the half-space

$$
\Omega=\{(x, z): x>0,-\infty<z<\infty\} .
$$

Along the boundary $x=0$ we require that

$$
u(0, z)= \begin{cases}1 & \text { for } z>0 \\ 0 & \text { for } z<0\end{cases}
$$

and far into the fresh-water region we impose the condition that

$$
v(x, z) \rightarrow 0 \text { as } z \rightarrow \infty \text { for every } x>0 .
$$

As with the classical Prandtl boundary layer in the wake of a flat plate, we construct the solution of (1.12) to (1.14) as a similarity solution. We introduce the stream function $\psi(x, z)$ :

$$
u=\frac{\partial \psi}{\partial z} \quad \text { and } \quad v=-\frac{\partial \psi}{\partial x}
$$

and write

$$
\psi(x, z)=\sqrt{ } x f(\eta), \quad \eta=z / \sqrt{ } x
$$

Then

$$
u=f^{\prime}(\eta) \text { and } v=\frac{\eta f^{\prime}(\eta)-f(\eta)}{2 \sqrt{x}},
$$

where the primes denote differentiation with respect to $\eta$. This shows that $(1.12)_{1}$ is satisfied for any $f \in C^{2}(\mathbb{R})$. Substitution into $(1.12)_{4}$ yields for $f$ the ordinary differential equation

$$
\left(2 \lambda f^{\prime}+\left|f^{\prime}\right| f^{\prime}\right)^{\prime \prime}+f f^{\prime \prime}=0 .
$$

Note that this equation resembles the Blasius equation $(8)$; however, it has the additional term $\left(\left|f^{\prime}\right| f^{\prime}\right)^{\prime \prime}$.

The conditions (1.13) at the boundary $x=0$ are satisfied if

$$
f^{\prime}(\eta) \rightarrow \begin{cases}1 & \text { if } \eta \rightarrow+\infty \\ 0 & \text { if } \eta \rightarrow-\infty\end{cases}
$$

and the condition (1.14) at $z=+\infty$ yields

$$
\eta f^{\prime}(\eta)-f(\eta) \rightarrow 0 \text { as } \eta \rightarrow+\infty
$$

In order to ensure that $v(x, z)$ remains bounded for each $x>0$, also as $z \rightarrow-\infty$, we replace $(1.16)_{2}$ by the stronger condition

$$
f(\eta) \rightarrow a \in \mathbb{R} \quad \text { as } \eta \rightarrow-\infty .
$$


Here the number $a$ is a priori not known but needs to be determined as part of the solution. We shall show in section 4 that $\eta f^{\prime}(\eta) \rightarrow 0$ as $\eta \rightarrow-\infty$. Hence

$$
\lim _{z \rightarrow-\infty} v(x, z)=-\frac{a}{2 \sqrt{ } x} .
$$

Thus, the number $a$ is directly related to the amount of salt drawn into the mixing zone. Therefore it is important to have some quantitative information about $a$.

In this paper it is assumed that

$$
f(\eta) \geqslant a \text { for all } \eta \in \mathbb{R} \text {. }
$$

In section 2 we show that this condition implies that solutions are monotonic so that $u=f^{\prime} \geqslant 0$, ruling out the possibility of back flow.

To study the problem posed by (1.15) to (1.19), we reduce (1.15) in section 2 to a second-order nonlinear diffusion equation, which we study in section 3 . In particular, existence and uniqueness of a solution is established for any $\lambda \geqslant 0$, subject to appropriate boundary conditions. This solution is then used in section 4 to obtain the unique solution $(a, f)$ of $(1.15)$ to $(1.19)$.

In addition to proving the existence and uniqueness of a solution we derive a number of qualitative properties of the function $f(\eta)$ and the limiting value $a$, in particular the dependence of the latter on $\lambda$. For $f$ we find that

$$
f(\eta)>\eta \text { for }-\infty<\eta<\infty
$$

and

(a) if $\lambda>0$, then

$$
f(\eta)>a, \quad f^{\prime}(\eta)>0, \quad f^{\prime \prime}(\eta)>0 \text { for }-x<\eta<x ;
$$

(b) if $\lambda=0$, then there exists a constant $x<0$ such that

$$
f(\eta)=a \text { for }-x<\eta \leqslant x
$$

and

$$
f(\eta)>a, \quad f^{\prime}(\eta)>0, \quad f^{\prime \prime}(\eta)>0 \text { for } x<\eta<x .
$$

A numerical computation shows that $x=-2 \cdot 4130$.

About the asymptotic behaviour of $f(\eta)$ as $\eta \rightarrow x$ and $\eta \rightarrow-x($ or $x)$ we show that

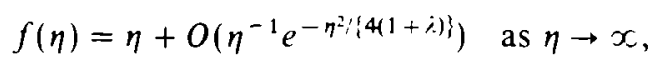

and

$$
\begin{array}{lll}
f(\eta)=a+O\left(e^{|a| \eta \mid 2 ;}\right) & \text { as } \eta \rightarrow-x & \text { if } i>0 \\
f(\eta)=a+\frac{1}{4}|a|(\eta-x)^{2}+O\left((\eta-x)^{3}\right) & \text { as } \eta \rightarrow x & \text { if } i=0
\end{array}
$$

For $a$ we find that it depends continuously and monotonically on $i$ and that 
it tends to $-\infty$ as $\lambda \rightarrow \infty$. Specifically, writing $a=a(\lambda)$ we prove that (i) for every $\lambda_{1}, \lambda_{2} \geqslant 0$ there exists a constant $C>0$ such that

$$
\left|a\left(\lambda_{1}\right)-a\left(\lambda_{2}\right)\right| \leqslant C\left|\lambda_{1}-\lambda_{2}\right|
$$

(ii) for every $\lambda_{1}, \lambda_{2} \geqslant 0$,

$$
\lambda_{1}>\lambda_{2} \Rightarrow a\left(\lambda_{1}\right)<a\left(\lambda_{2}\right)
$$

and we prove the following upper and lower bounds:

$$
-a^{*} \sqrt{ }(\lambda+1)<a(\lambda)<-a^{*} \sqrt{ } \lambda \text { for } \lambda \geqslant 0,
$$

where $a^{*}=1 \cdot 23675$. In Table 1 we give some values of $a$.

$\begin{array}{rc} & \text { Table 1 } \\ \lambda & a(\lambda) \\ & \\ 0 & -0.7102 \\ 1 & -1.4256 \\ 2 & -1.8885 \\ 10 & -4.0013\end{array}$

Finally, in section 5 we consider the case when both the fresh water (far up) and the salt water (far down) move in the positive $x$-direction:

$$
u(x, z) \rightarrow \begin{cases}U_{f} & \text { as } z \rightarrow \infty, \\ U_{s} & \text { as } z \rightarrow-\infty\end{cases}
$$

where $U_{f}=U_{s}+U$ and $U_{s}>0$. This leads again to equation (1.15) and condition (1.17). However, conditions $(1.16)_{1.2}$ become

$$
f^{\prime}(\eta)= \begin{cases}1+\theta & \text { if } \eta \rightarrow \infty \\ \theta & \text { if } \eta \rightarrow-\infty\end{cases}
$$

where $\theta=U_{s} / U$.

\section{Preliminaries}

In this section we shall establish some qualitative properties of solutions. It will be convenient to rewrite the problem slightly. Set

$$
g(s)=2 i s+|s| s, \quad \lambda \geqslant 0 .
$$

Then we shall consider the problem

$$
\begin{gathered}
\left(g\left(f^{\prime}\right)\right)^{\prime \prime}+f f^{\prime \prime}=0 \quad \text { on }(-\infty, \infty), \\
f(-\infty)=a \quad \text { and } \quad f^{\prime}(\infty)=1, \\
\lim _{\eta \rightarrow \infty}\left\{\eta f^{\prime}(\eta)-f(\eta)\right\}=0,
\end{gathered}
$$


where $a \in \mathbb{R}$. By a solution of (2.1) to (2.3) we shall mean a number $a$ and a function $f: \mathbf{R} \rightarrow[a, \infty)$ such that (i) both $f^{\prime}$ and $\left(g\left(f^{\prime}\right)\right)^{\prime}$ are locally absolutely continuous on $\mathbb{R}$, (ii) equation (2.1) is satisfied almost everywhere and (iii) (2.2) and (2.3) hold.

Suppose that $f$ is a solution of $(2.1)$ to (2.3). Then we can define the set $I$ on which $f^{\prime}>0$ :

$$
I=\left\{\eta \in \mathbb{R}: f^{\prime}(\eta)>0\right\} .
$$

In view of the condition at infinity, $I$ is non-empty. It will also be convenient to introduce the function $F$ defined by

$$
F(\eta)=g\left(f^{\prime}(\eta)\right), \quad-\infty<\eta<\infty .
$$

Equation (2.1) can then be written as

$$
F^{\prime \prime}+f f^{\prime \prime}=0 \text {. }
$$

Lemma 2.1. $f \in C^{\infty}(I)$. If $\lambda>0$ then $f \in C^{2}(\mathbb{R})$.

Proof. The function $g$ satisfies $g^{\prime}(s)>g^{\prime}(0)=2 \lambda$. for $s \in \mathbb{R} \backslash\{0\}$ and by the definition of a solution, $F^{\prime}$ is locally absolutely continuous on $\mathbb{R}$. Thus, since

$$
f^{\prime}(\eta)=g^{-1}(F(\eta)) \text { for } \eta \in \mathbb{R}
$$

it follows that $f \in C^{2}(I)$ or, if $\lambda>0$, that $f \in C^{2}(\mathbb{R})$. The first assertion now follows from a standard bootstrap argument.

LEMMA 2.2. We have

$$
f^{\prime}(\eta) \rightarrow 0 \text { and } F^{\prime}(\eta) \rightarrow 0 \text { as } \eta \rightarrow-\infty \text {. }
$$

Proof. We first write equation (2.1) as

$$
\left(2 \lambda f^{\prime}+\left|f^{\prime}\right| f^{\prime}+\frac{1}{2} f^{2}\right)^{\prime \prime}=f^{\prime 2} \geqslant 0 \quad \text { a.e. on }(-\infty, \infty) \text {. }
$$

This shows that the function

$$
h(\eta)=2 \lambda f^{\prime}(\eta)+\left|f^{\prime}(\eta)\right| f^{\prime}(\eta)+\frac{1}{2} f^{2}(\eta)
$$

is convex on $\mathbb{R}$. We assert that

$$
h \geqslant 0 \text { and } h^{\prime} \geqslant 0 \text { on }(-\infty, \infty) \text {. }
$$

To prove that $h$ is non-decreasing, suppose that, for some $\eta_{0} \in \mathbb{R}, h^{\prime}\left(\eta_{0}\right)<0$. Then by the convexity of $h$,

$$
h(\eta) \rightarrow \infty \quad \text { as } \eta \rightarrow-\infty .
$$

Since, by (2.2), $f(\eta)$ tends to some constant as $\eta \rightarrow-\infty,(2.7)$ implies that $f^{\prime}(\eta)$ becomes unbounded, violating this boundary condition.

Next, suppose that, for some $\eta_{0} \in \mathbb{R}, h\left(\eta_{0}\right)<0$. Then by the previous result 
$h(\eta)<0$ for all $\eta \leqslant \eta_{0}$ and hence

$$
2 \lambda f^{\prime}+\left|f^{\prime}\right| f^{\prime} \leqslant 2 \lambda f^{\prime}+\left|f^{\prime}\right| f^{\prime}+\frac{1}{2} f^{2}<0 \quad \text { on }\left(-\infty, \eta_{0}\right] .
$$

This implies that $f^{\prime}(\eta)<-\delta<0$ for some $\delta>0$ for all $\eta \in\left(-\infty, \eta_{0}\right]$, and thus that $f(\eta) \rightarrow-\infty$ as $\eta \rightarrow-\infty$, violating the boundary condition.

It follows from (2.6) that

$$
\lim _{\eta \rightarrow-\infty} h(\eta) \text { exists }=b \geqslant 0 .
$$

This implies, by the boundary condition at $\eta=-\infty$, that

$$
\lim _{\eta \rightarrow-\infty}\left\{2 \lambda f^{\prime}(\eta)+\left|f^{\prime}(\eta)\right| f^{\prime}(\eta)\right\}=\frac{1}{2} a^{2}-b,
$$

and hence that

$$
\lim _{\eta \rightarrow-\infty} f^{\prime}(\eta) \text { exists }=c .
$$

Plainly, by the boundary condition, $c=0$, so that by (2.9), $b=\frac{1}{2} a^{2}$.

To prove the second assertion of Lemma 2.2, we use the fact that by the convexity of $h$,

$$
\lim _{\eta \rightarrow-\infty} h^{\prime}(\eta) \text { exists }=d
$$

where $d \geqslant 0$ in view of (2.6). If $d>0$ then $h(\eta) \rightarrow-\infty$ as $\eta \rightarrow-\infty$, which is impossible, because $h(\eta) \geqslant 0$ for all $\eta \in \mathbb{R}$. Therefore $d=0$, and we have

$$
\lim _{\eta \rightarrow-\infty} h^{\prime}(\eta)=0
$$

Because

$$
F^{\prime}(\eta)=h^{\prime}(\eta)-f(\eta) f^{\prime}(\eta)
$$

we conclude using (2.10) and the boundary condition that

$$
\lim _{\eta \rightarrow-\infty} F^{\prime}(\eta)=0
$$

as well.

LEMMA 2.3. We have

$$
f^{\prime}(\eta)>0 \text { if } f(\eta)>a .
$$

Proof. When we integrate equation (2.1) over $(-\infty, \eta)$ and use Lemma 2.2, we obtain

$$
F^{\prime}(\eta)+f(\eta) f^{\prime}(\eta)=\int_{-\infty}^{\pi}\left\{f^{\prime}(s)\right\}^{2} d s
$$

Suppose that there exists a point $\eta_{1} \in \mathbb{R}$ such that both $f\left(\eta_{1}\right)>a$ and 
$f^{\prime}\left(\eta_{1}\right) \leqslant 0$. Then the boundary condition $f(-\infty)=a$ implies the existence of a point $\eta_{0} \in\left(-\infty, \eta_{1}\right]$ such that

$$
f\left(\eta_{0}\right)>a, \quad f^{\prime}\left(\eta_{0}\right)=0
$$

and

$$
f(\eta) \leqslant f\left(\eta_{0}\right) \text { for }-\infty<\eta \leqslant \eta_{0} .
$$

Thus at $\eta=\eta_{0}(2.12)$ becomes

$$
\left(2 \lambda f^{\prime}+\left|f^{\prime}\right| f^{\prime}\right)^{\prime}\left(\eta_{0}\right)=\int_{-\infty}^{\eta_{0}}\left\{f^{\prime}(s)\right\}^{2} d s .
$$

If $\lambda>0$, then by Lemma $2.1, f$ is smooth in a neighbourhood of $\eta_{0}$. Thus, in this case

$$
f^{\prime \prime}\left(\eta_{0}\right)>0
$$

which implies that $f(\eta)>f\left(\eta_{0}\right)$ in a left neighbourhood of $\eta_{0}$, contradicting (2.13).

If $\lambda=0$, we conclude from (2.14) that

$$
\left(\left|f^{\prime}\right| f^{\prime}\right)^{\prime}\left(\eta_{0}\right)>0 \text {. }
$$

Since by the definition of a solution, $\left|f^{\prime}\right| f^{\prime} \in C^{1}(\mathbb{R})$, it follows that $\left(\left|f^{\prime}\right| f^{\prime}\right)^{\prime}>0$ in a neighbourhood of $\eta_{0}$. This implies that $\left|f^{\prime}\right| f^{\prime}<0$ in a left neighbourhood of $\eta_{0}$ and thus that $f(\eta)>f\left(\eta_{0}\right)$ in this neighbourhood, again contradictiing (2.13).

By definition $f \geqslant a$ on $\mathbb{R}$. Hence Lemma 2.3 implies that

$$
f^{\prime} \geqslant 0 \text { on } \mathbb{R} \text {. }
$$

This allows us to reformulate the result of Lemma 2.1 .

Corollary 2.4. (a) If $\lambda>0$ then $f \in C^{\infty}(\mathbb{R})$;

(b) if $\lambda=0$ then $f \in C^{x}(\{f>a\})$.

Define

$$
\alpha=\inf \{\eta \in \mathbb{R} ; f(\eta)>a\} .
$$

Plainly, by Lemma 2.3,

$$
f>a \text { and } f^{\prime}>0 \text { on }(\alpha, \infty) .
$$

Near $\eta=\alpha$ we have

$$
\lim _{\eta ! x} f(\eta)=a
$$


by the condition at $\eta=-\infty$ and the continuity of $f$, and

$$
\lim _{\eta \downarrow \alpha} f^{\prime}(\eta)=0, \quad \lim _{\eta \downarrow \alpha} F^{\prime}(\eta)=0
$$

by Lemma 2.2 and the continuity of $f^{\prime}$ and $F^{\prime}$.

Rather than studying the problem (2.1) to (2.3) as it stands, we first transform it, in a way well known in the theory of the Falkner-Skan equation (9) to a problem involving a second-order equation. Thus, we introduce $f$ as an independent variable, which is possible by Lemma 2.3 , and choose $f^{\prime}$ as a dependent variable. Specifically, define $\sigma:(a, \infty) \rightarrow(\alpha, \infty)$ by

$$
\sigma(f(\eta))=\eta \quad \text { for } \alpha<\eta<\infty
$$

and $y:[a, \infty) \rightarrow(\alpha, \infty)$ by

$$
y(f)= \begin{cases}f^{\prime}(\sigma(f)) & \text { if } a<f<\infty, \\ 0 & \text { if } f=a .\end{cases}
$$

In view of the continuity of $f^{\prime}$ and Corollary $2.4, y \in C([a, \infty)) \cap C^{\infty}(a, \infty)$.

For $f>a$ or $\eta>\alpha$, we have

$$
f^{\prime}(\eta)=y(f(\eta))
$$

and so, upon differentiation we obtain

$$
f^{\prime \prime}(\eta)=y^{\prime}(f(\eta)) y(f(\eta))
$$

Similarly,

$$
\left(g\left(f^{\prime}\right)\right)^{\prime}=g^{\prime}(y) y^{\prime} y
$$

and

$$
\left.\left(g\left(f^{\prime}\right)\right)\right)^{\prime \prime}=\left(y g^{\prime}(y) y^{\prime}\right)^{\prime} y .
$$

When we substitute this into (2.1) and use Lemma 2.3, we arrive at the equation

$$
\left(\left(\lambda y+y^{2}\right) y^{\prime}\right)^{\prime}+\frac{1}{2} f y^{\prime}=0 \quad \text { when } a<f<\infty,
$$

whilst (2.2) becomes

$$
y(f) \rightarrow 1 \text { as } f \rightarrow \infty .
$$

Finally, from (2.16) and (2.17) we conclude that

$$
y(f) \rightarrow 0 \text { and }\left\{\lambda y(f)+y^{2}(f)\right\} y^{\prime}(f) \rightarrow 0 \quad \text { as } f \rightarrow a .
$$

Thus, we have shown that if $(a, f)$ is a solution of $(2.1)$ and $(2.2)$ then $\left(a, f^{\prime}(\sigma(f))\right)$ is a solution of $(2.20),(2.21)$ and $(2.22)$.

In the next section we shall show that the problem $(2.20)$ to $(2.22)$ has a unique solution $(a, y)$.

Having obtained $a$ and $y(f)$, and therefore also $f^{\prime}(\eta)$, we can obtain $f(\eta)$ by integration. This involves a constant of integration. We shall show that this 
constant can be chosen so that the condition (2.3) at $\eta=\infty$ is satisfied, and that this choice is unique.

\section{The diffusion problem}

In section 2 we transformed the original problem (2.1) to (2.3) involving a third-order ordinary differential equation for $f(\eta)$ to one with a second-order ordinary differential equation for $y(f)=f^{\prime}(\sigma(f))$. In this section we study the latter problem. It is of the form

$$
\left\{\begin{array}{l}
\left(D(y) y^{\prime}\right)^{\prime}+\frac{1}{2} f y^{\prime}=0, \quad a<f<\infty, \\
y(a)=0, \quad\left(D(y) y^{\prime}\right)(a)=0, \\
y(f) \rightarrow 1 \quad \text { as } f \rightarrow \infty,
\end{array}\right.
$$

where

$$
D(s)=\lambda s+s^{2} .
$$

Note that if $y$ is a solution of (3.1) to (3.3), then $y^{\prime}>0$ on $(a, \infty)$ and $y^{\prime \prime}<0$ on $(0, \infty)$, and so $y^{\prime}(f) \rightarrow 0$ as $f \rightarrow \infty$. In Lemma 3.5 we shall establish the rate of convergence.

Problem (I) is a classical problem, which arises in the study of similarity solutions of the nonlinear diffusion equation

$$
u_{t}=\left(D(u) u_{x}\right)_{x} .
$$

Since the diffusion coefficient $D(s)$ vanishes at $s=0$, and in fact

$$
\int_{0} \frac{D(s)}{s} d s<\infty
$$

equation (3.5) is degenerate and gives rise to free boundaries. For further references we refer to $(10,11)$.

In analysing Problem (I) we follow in particular the theory developed in (12 to 15). There, we often work with $y$ as the independent variable. This is possible because $y$ is strictly monotonic on $[a, \infty)$. Thus, define $\tau:[0,1) \rightarrow[a, \infty)$ by

$$
\tau(y(f))=f, \quad a \leqslant f<\infty .
$$

As the new dependent variable we choose

$$
z(y)=-\int_{0}^{y} \tau(\xi) d \xi .
$$

To derive an equation for $z$, we integrate (3.1) over $(a, f)$. If we then use the continuity of the flux $D(y) y^{\prime}$ at $f=a$, we obtain

$$
D(y) y^{\prime}=-\frac{1}{2} \int_{a}^{f} s y^{\prime}(s) d s=-\frac{1}{2} \int_{0}^{y} \tau(\xi) d \xi,
$$


and so

$$
z=2 D(y) y^{\prime}
$$

On the other hand, when we differentiate (3.6) twice we obtain

$$
z^{\prime \prime}=-1 / y^{\prime}
$$

When we now use (3.7) to eliminate $y^{\prime}$ from (3.8) we obtain the equation

$$
z z^{\prime \prime}=-2 D(y), \quad 0<y<1 .
$$

Consistent with the conditions imposed on $y(f)$ at $f=a$ and $f=\infty$, we choose at the end points $y=0$ and $y=1$,

$$
z(0)=0 \text { and } z(1)=0 .
$$

Finally, by (3.6) and (3.3),

$$
z^{\prime}(1)=-\infty \text {. }
$$

Thus, we have arrived at the following problem

$$
\left\{\begin{array}{l}
z z^{\prime \prime}=-2 D(y), \quad 0<y<1, \\
z(0)=0, \quad z(1)=0, \quad z^{\prime}(1)=-\infty,
\end{array}\right.
$$

and we seek a solution $z \in C^{2}(0,1) \cap C([0,1])$. The existence and uniqueness of such a solution was established in $(14,15)$. This in turn implies existence and uniqueness for Problem (I).

Let $z$ be the solution of (II). Then, because $D>0$ on $(0,1), z$ and $z^{\prime \prime}$ have one sign on $(0,1)$. Since $z^{\prime}(1)=-\infty$, it follows that $z^{\prime \prime}<0$ on $(0,1)$ and so, by the differential equation, $z>0$ on $(0,1)$. Therefore

$$
\lim _{y \rightarrow 0}-z^{\prime}(y) \text { exists }=a<0 \text {. }
$$

Also

$$
a>-\infty \text {. }
$$

To see this, we write the differential equation as

$$
\frac{D(y)}{y}=-\frac{1}{2} \frac{z}{y} z^{\prime \prime} .
$$

By the convexity of $z$, we have $z / y>z^{\prime}$ and hence

$$
\frac{D(y)}{y}>-\frac{1}{2} z^{\prime} z^{\prime \prime}
$$

which yields upon integration over $\left(0, y_{0}\right)$, where $y_{0} \in(0,1)$ is the zero of $z^{\prime}$,

$$
\int_{0}^{y_{0}} \frac{D(s)}{s} d s>\frac{1}{4} z^{\prime 2}(0)
$$


and hence

$$
a>-2\left(\int_{0}^{y_{0}} \frac{D(s)}{s}\right)^{\frac{1}{2}} .
$$

Note that $y_{0}=y(0)$.

Summarizing we have proved the following result.

Theorem 3.1. Problem (I) has a unique solution ( $a, y)$. Moreover,

$$
-2\left(\int_{0}^{y(0)} \frac{D(s)}{s} d s\right)^{\frac{1}{2}}<a<0 .
$$

We note a monotonicity property of $a$ with respect to $\lambda_{n}$ Let $\lambda_{1}<\lambda_{2}$ and set

$$
D_{i}(y)=\lambda_{i} y+y^{2} \quad(i=1,2) .
$$

Then, plainly, $D_{1}(y)<D_{2}(y)$ for $0<y \leqslant 1$. Suppose that $z_{1}$ and $z_{2}$ are the solutions of (II) which correspond respectively to $D_{1}$ and $D_{2}$. Then by $(14$, Theorem $2.1 ; 15$, Theorem 4),

$$
z_{1}<z_{2} \text { on }(0,1) \text { and } z_{1}^{\prime}(0)<z_{2}^{\prime}(0) \text {. }
$$

Thus, if we write $a=a(\lambda)$, emphasizing the dependence on $\lambda$, we have the following monotonicity property.

TheOrem 3.2. Let $a(\lambda)$ be as defined above. Then

$$
\lambda_{1}<\lambda_{2} \Rightarrow a\left(\lambda_{1}\right)>a\left(\lambda_{2}\right) \text {. }
$$

In the next theorem we show that $a(\lambda)$ is locally Lipschitz continuous for $0 \leqslant \lambda<\infty$.

THEOREM 3.3. For every $\lambda_{1}, \lambda_{2} \geqslant 0$ there exists a constant $\mathscr{C}(\bar{\lambda})$, where $\bar{\lambda}=$ $\min \left\{\lambda_{1}, \lambda_{2}\right\}$, such that

$$
\left|a\left(\lambda_{1}\right)-a\left(\lambda_{2}\right)\right|<\mathscr{B}(\bar{\lambda})\left|\lambda_{1}-\lambda_{2}\right| .
$$

Proof. Let $\lambda_{1}, \lambda_{2} \geqslant 0$ and let $z_{1}$ and $z_{2}$ be the solutions of Problem (II) corresponding respectively to $\lambda_{1}$ and $\lambda_{2}$. Without loss of generality we choose $\lambda_{2}>\lambda_{1} \geqslant 0$. Set

$$
v=z_{2}-z_{1} .
$$

Then $v \in C^{2}(0,1) \cap C([0,1]), v>0$ on $(0,1)$ and $v(0)=v(1)=0$. Therefore, $v$ attains its maximum value at a point $b \in(0,1)$ :

$$
v(b)>0, \quad v^{\prime}(b)=0, \quad v^{\prime \prime}(b) \leqslant 0 .
$$

According to the differential equation for $z$,

$$
-z_{2} z_{2}^{\prime \prime}+z_{1} z_{1}^{\prime \prime}=2\left(\lambda_{2}-\lambda_{1}\right) y
$$


or

$$
\left(z_{2}-z_{1}\right)\left|z_{1}^{\prime \prime}\right|=2\left(\lambda_{2}-\lambda_{1}\right) y+z_{2} v^{\prime \prime} .
$$

Thus, at $y=b$ we have

$$
\begin{aligned}
\left(z_{2}-z_{1}\right)(b) & \leqslant 2\left(\lambda_{2}-\lambda_{1}\right) \frac{b}{\left|z_{1}^{\prime \prime}(b)\right|}=\left(\lambda_{2}-\lambda_{1}\right) z_{1}(b) \frac{b}{D_{1}(b)} \\
& \leqslant\left(\lambda_{2}-\lambda_{1}\right) \frac{z_{1}(b)}{b}<\left(\lambda_{2}-\lambda_{1}\right) z_{1}^{\prime}(0)
\end{aligned}
$$

and so

$$
\left\|z_{2}-z_{1}\right\|_{\infty}<\left(\lambda_{2}-\lambda_{1}\right) z_{1}^{\prime}(0) \text {. }
$$

When we divide (3.12) by $z_{1}$ and recall that $z_{1}<z_{2}$, we obtain

$$
-v^{\prime \prime}(y)<2\left(\lambda_{2}-\lambda_{1}\right) \frac{y}{z_{1}(y)} .
$$

To remove the singularity at $y=1$ we multiply by $1-y$. Then

$$
-v^{\prime \prime}(y)(1-y)<2\left(\lambda_{2}-\lambda_{1}\right) \frac{y(1-y)}{z_{1}(y)}<K\left(\lambda_{2}-\lambda_{1}\right),
$$

where $K$ is a positive generic constant, because of the concavity of $z_{1}$. This yields upon integration over $(0, b)$

$$
-\left.v^{\prime}(y)(1-y)\right|_{0} ^{b}-\left.v(y)\right|_{0} ^{b}<K\left(\lambda_{2}-\lambda_{1}\right)
$$

or

$$
v^{\prime}(0)<K\left(\hat{\lambda}_{2}-\lambda_{1}\right)+v(b) .
$$

Thus, in view of (3.13) there exists a positive constant $\mathscr{B}$ such that

$$
v^{\prime}(0)<\mathscr{C}\left(\lambda_{2}-\lambda_{1}\right)
$$

Since $v^{\prime}(0)=z_{2}^{\prime}(0)-z_{1}^{\prime}(0)=-a\left(\lambda_{2}\right)+a\left(\lambda_{1}\right)$ the assertion follows.

Finally, using a comparison argument based on the inequalities

$$
\lambda y<D(y)<(\lambda+1) y \text { if } 0<y<1 \text {, }
$$

and using a scaling property of equation (3.1) with $D(y)=y$, we obtain the following bounds for $a(\lambda)(3)$.

TheOREM 3.4. Let $a(\lambda)$ be as defined above. Then

$$
-a^{*} \sqrt{ }(\lambda+1)<a(i)<-a^{*} \sqrt{ } \lambda \text { for } i \geqslant 0
$$

where $a^{*}=1 \cdot 23675$. 
COROllary 3.5. $a(\lambda) \rightarrow-\infty$ as $\lambda \rightarrow \infty$.

For later use we need an estimate for the rate with which $y(f)$ approaches the value 1 as $f$ tends to infinity.

LEMMA 3.6. We have

$$
y(f)=1+O\left(f^{-1} e^{-\gamma f^{2}}\right) \text { and } y^{\prime}(f)=O\left(e^{-\gamma f^{2}}\right) \text { as } f \rightarrow \infty .
$$

where $\gamma=1 /\{4(1+i)\}$.

Proof. Define the function

$$
w(s)=\int_{0}^{y(s)} D(t) d t
$$

This satisfies the equation

$$
w^{\prime \prime}+\frac{f}{2 D(y)} w^{\prime}=0
$$

and so, since $w^{\prime}=D(y) y^{\prime}>0$ on $(a, \infty)$,

$$
\log w^{\prime}(f)=-\frac{1}{2} \int^{s} \frac{s}{D(y(s))} d s+\text { constant. }
$$

But $y(f)<1$ and so $D(y(f))<D(1)$ for all $f \in(a, \infty)$. Therefore

$$
\log w^{\prime}(f)<-\frac{f^{2}}{4 D(1)}+\text { constant }
$$

or

$$
w^{\prime}(f)<C_{1} \exp \left(-\frac{f^{2}}{4 D(1)}\right), \quad a<f<\infty,
$$

where $C_{1}$ is a positive constant. Because $D(y)>D\left(\frac{1}{2}\right)$ for $f$ large enough this means that

$$
y^{\prime}(f)<C_{2} \exp \left(-\frac{f^{2}}{4 D(1)}\right) \text { for } f \text { large enough, }
$$

where $C_{2}=C_{1} / D\left(\frac{1}{2}\right)$. The assertion now follows upon integration.

\section{The solution $f(\eta)$}

From the solution $y(f)$ obtained in section 3 we now construct the solution $f(\eta)$ of the original problem (2.1) to (2.3). In view of its definition (2.19),y yields the first-order differential equation

$$
\frac{d \eta}{d f}=\frac{1}{y(f)}, \quad a<f<\infty
$$


where we have replaced $\sigma$ by $\eta$ for the sake of clarity. Remembering that $a<0$, we can integrate $(4.1)$ over $(0, f)$ with $f>a$. This yields

$$
\eta(f)=\eta_{0}+\int_{0}^{f} \frac{d s}{y(s)}=\eta_{0}+f+\int_{0}^{f} \frac{1-y(s)}{y(s)} d s,
$$

where $\eta_{0}$ is a constant which we are still free to choose. We shall use this freedom to satisfy the second boundary condition at infinity:

$$
\lim _{\eta \rightarrow \infty}\left\{\eta f^{\prime}(\eta)-f(\eta)\right\}=0
$$

By Lemma 3.6 the integrand $(1-y) / y$ in $(4.2)$ is integrable over $(0, \infty)$ and

$$
\int_{s}^{\infty} \frac{1-y(s)}{y(s)} d s=O\left(f^{-2} e^{-\gamma f^{2}}\right) \text { as } f \rightarrow \infty .
$$

Thus, we can write (4.2) as

$$
\eta(f)=f+\eta_{0}+A+O\left(f^{-2} e^{-y f^{2}}\right) \text { as } f \rightarrow \infty,
$$

where

$$
A=\int_{0}^{\infty} \frac{1-y(s)}{y(s)} d s .
$$

Note that since $y(f)$ is an increasing function which tends to 1 as $f \rightarrow \infty$, it follows that $y(f)<1$ for all $f \in(a, \infty)$ and so $A>0$.

Turning to the boundary condition (4.3), we note that

$$
\eta f^{\prime}-f=\eta(f) y(f)-f .
$$

With the expression (4.5) for $\eta(f)$ we now write the right-hand side of $(4.7)$ as

$$
\begin{aligned}
\eta(f) y(f)-f & =f\{y(f)-1\}+\left\{A+\eta_{0}+O\left(f^{-2} e^{-\gamma f^{2}}\right)\right\} y(f) \\
& =A+\eta_{0}+O\left(e^{-\gamma f^{2}}\right) \text { as } f \rightarrow \infty,
\end{aligned}
$$

where we have used Lemma 3.6 once more. Therefore, in order to satisfy (4.3) we need to choose

$$
\eta_{0}=-\int_{0}^{\infty} \frac{1-y(s)}{y(s)} d s .
$$

Note that $\eta_{0}$ is uniquely determined by $y$.

For the inverse $\eta(f)$ of the solution we thus find from (4.2) that

$$
\eta(f)=f-\int_{f}^{\infty} \frac{1-y(s)}{y(s)} d s .
$$

This completes the construction of the solution of the problem (2.1) to (2.3).

We conclude this section with a few observations about the solution $f(\eta)$. 
From (4.9) we deduce that

$$
\eta(f)<f \text { for } a<f<\infty
$$

which implies that

$$
f(\eta)>\eta \text { for } \alpha<\eta<\infty,
$$

where $\alpha=\lim _{f \rightarrow a^{+}} \eta(f)$ was defined in section 2 . In view of the asymptotic behaviour of $\eta(f)$ as $f \rightarrow \infty$ and (4.9) we find that

$$
f(\eta)=\eta+O\left(\eta^{-2} e^{-\eta^{2} /\{4(1+\lambda)\}}\right) \text { as } \eta \rightarrow \infty .
$$

For $\alpha$ we find from (4.8) that

$$
\alpha=a-\lim _{s \rightarrow a^{+}} \int_{f}^{\infty} \frac{1-y(s)}{y(s)} d s .
$$

Since $a<0$ it is clear that $\alpha<0$. Depending on the behaviour of $y(f)$ near $f=a, \alpha$ will be bounded or unbounded.

To determine the behaviour of $y(f)$ near $f=a$ we proceed as in (12) and integrate (3.1) over $(a, f)$, where $a<f<0$. This yields

$$
D(y(f)) y^{\prime}(f)=\frac{1}{2} \int_{0}^{f}\left|s y^{\prime}(s)\right| d s,
$$

or, writing $f=\tau(y)$ as in section 3 ,

$$
\frac{d \tau}{d y}=\frac{2 D(y)}{\int_{0}^{y}|\tau(s)| d s} .
$$

Since $\tau(y)=a+o(1)$ as $y \rightarrow 0$, we obtain upon integration that

$$
\tau(y)=a+2 \int_{0}^{y} \frac{D(t) d t}{\int_{0}^{t}|\tau(s)| d s} .
$$

Clearly

$$
\int_{0}^{t}|\tau(s)| d s=|a| t[1+o(1)] \quad \text { as } t \rightarrow 0
$$

and hence by (4.12)

$$
\tau(y)=a+\frac{2}{|a|} \int_{0}^{y} \frac{D(t)}{t} d t[1+o(1)] \text { as } y \rightarrow 0
$$

which implies that

$$
\tau(y)=a+o\left(\lambda y+y^{2}\right) \text { as } y \rightarrow 0 .
$$


Thus, we can strengthen (4.13) to

$$
\int_{0}^{t}|\tau(s)| d s=|a| t\left[1+o\left(\lambda t+t^{2}\right)\right] \quad \text { as } t \rightarrow 0
$$

which yields in (4.12)

$$
\tau(y)=a+\frac{2}{|a|} \int_{0}^{y} \frac{D(t)}{t} d t\left[1+o\left(\lambda y+y^{2}\right)\right] \quad \text { as } y \rightarrow 0,
$$

or

$$
\tau(y)=a+\frac{2}{|a|}\left(\lambda y+\frac{1}{2} y^{2}\right)\left[1+o\left(\lambda y+y^{2}\right)\right] \quad \text { as } y \rightarrow 0 .
$$

Thus,

$$
\begin{aligned}
& \tau(y)=a+\frac{2 \lambda}{|a|} y+O\left(y^{2}\right) \quad \text { as } y \rightarrow 0 \quad \text { if } \lambda>0, \\
& \tau(y)=a+\frac{1}{|a|} y^{2}+O\left(y^{4}\right) \quad \text { as } y \rightarrow 0 \quad \text { if } \lambda=0 .
\end{aligned}
$$

We conclude that, as $f \rightarrow a^{+}$,

$$
y(f)= \begin{cases}\frac{|a|}{2 \lambda}(f-a)[1+O(f-a)] & \text { if } \lambda>0, \\ \sqrt{ }\{|a|(f-a)\}[1+O(\sqrt{ }\{f-a\})] & \text { if } \lambda=0 .\end{cases}
$$

Using this in the expression (4.11) for $\alpha$ we find that

$$
\begin{aligned}
\alpha=-\infty & \text { if } \lambda>0, \\
-\infty<\alpha<0 & \text { if } \lambda=0 .
\end{aligned}
$$

The behaviour of $f(\eta)$ near $\eta=\alpha$ can now be obtained from (4.1).

For $\lambda>0$ we obtain

$$
\frac{d \eta}{d f}=\frac{2 \lambda}{|a|} \frac{1}{f-a}[1+O(f-a)] \text { as } f \rightarrow a,
$$

so that

$$
\eta(f)=\frac{2 \lambda}{|a|} \log (f-a)+O(1) \text { as } f \rightarrow a,
$$

or

$$
f(\eta)-a=O\left(e^{|a| \eta / 2 j}\right) \quad \text { as } \eta \rightarrow-\infty
$$


For $\lambda=0$ we obtain in a similar fashion

$$
f(\eta)-a=\frac{1}{4}|a|(\eta-\alpha)^{2}+O\left((\eta-\alpha)^{3}\right) \quad \text { as } \eta \rightarrow \alpha .
$$

Finally we observe that since $y^{\prime}>0$ in $(a, \infty)$, we have in both cases

$$
f^{\prime \prime}(\eta)>0 \text { if } f(\eta)>a .
$$

The qualitative properties obtained in this section and in the previous one are clearly demonstrated in Fig. 2, where we present the numerical solution of Problem (II) and the corresponding solution of the problem (2.1) to (2.3) for different values of $\lambda$.

\section{Non-stagnant salt water}

Here we consider the case when initially, at $x=0$, the fresh and the salt water are both moving in the positive $x$-direction. Thus the boundary condition at $x=0$ is replaced by

$$
u(0, z)= \begin{cases}U_{f} & \text { for } z<0, \\ U_{s} & \text { for } z<0,\end{cases}
$$

where $U_{f}=U_{s}+U$ and $U_{s}>0$. Looking again for a similarity solution, as we did previously, we arrive at a boundary-value problem consisting of equation (1.15) together with the boundary conditions (1.17) and (1.21). Instead of (1.19) we now require that solutions are strictly increasing:

$$
f^{\prime}(\eta)>0 \text { for all } \eta \in \mathbb{R} \text {. }
$$

Solving this problem leads to a velocity field in which the horizontal component $u$ satisfies $(1.20)$.

Again, we introduce the variable $y=y(f)$ for which we find the problem

$$
\left\{\begin{array}{l}
\left.D(y) y^{\prime}\right)^{\prime}+\frac{1}{2} f y^{\prime}=0 \text { for }-\infty<f<\infty, \\
y(-\infty)=\theta, \quad y(\infty)=1+\theta
\end{array}\right.
$$

where $\theta=U_{s} / U$. Using the results of (15) and a bootstrap argument we obtain that for each $\lambda \geqslant 0$, Problem (III) has a unique solution $y \in C^{\infty}(\mathbb{R})$. This solution satisfies

$$
y^{\prime}(f)>0 \text { for all } f \in \mathbb{R} .
$$

With respect to the asymptotic behaviour at $-\infty$ and $+\infty$ we find, as in Lemma 3.6, that

$$
\begin{aligned}
& y(f)=\theta+O\left(f^{-1} e^{-\gamma f^{2}}\right) \text { as } f \rightarrow-\infty \\
& y(f)=1+\theta+O\left(f^{-1} e^{-\gamma+f^{2}}\right) \text { as } f \rightarrow+\infty
\end{aligned}
$$

where

$$
\gamma_{-}=\frac{1}{4\left(\lambda \theta+\theta^{2}\right)} \text { and } \gamma_{+}=\frac{1}{4\left\{\lambda(1+\theta)+(1+\theta)^{2}\right\}} \text {. }
$$



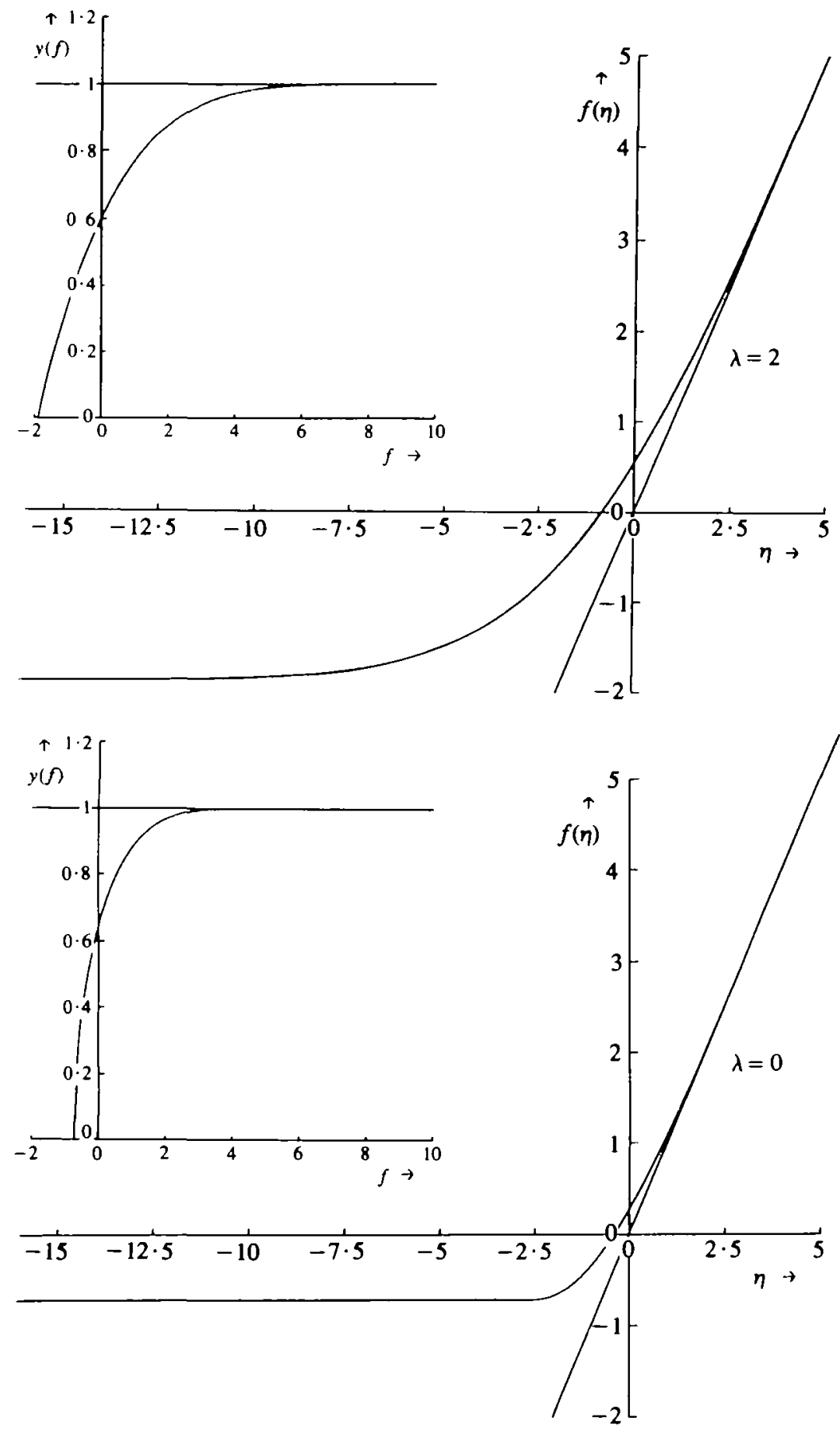

FIG. 2. Graphs of $y(f)$ and $f(\eta)$ 
To obtain from $y=y(f)$ the solution $f=f(\eta)$ we follow the procedure of section 4 . We now write

$$
\begin{aligned}
\eta(f) & =\eta_{0}+\int_{0}^{f} \frac{d s}{y(s)} \\
& =\eta_{0}+\frac{f}{1+\theta}+\frac{1}{1+\theta} \int_{0}^{s} \frac{1+\theta-y(s)}{y(s)} d s
\end{aligned}
$$

or

$$
\eta(f)=\eta_{0}+\frac{f}{1+\theta}+A-\frac{1}{1+\theta} \int_{f}^{\infty} \frac{1+\theta-y(s)}{y(s)} d s,
$$

where

$$
A=\frac{1}{1+\theta} \int_{0}^{\infty} \frac{1+\theta-y(s)}{y(s)} d s
$$

Again we set

$$
A+\eta_{0}=0
$$

to ensure that

$$
\eta f^{\prime}(\eta)-f(\eta)=\eta(f) y(f)-f=O\left(e^{-\gamma+\delta^{2}}\right) \text { as } f \rightarrow \infty,
$$

so that (1.17), the second boundary condition at $+\infty$, is satisfied. Therefore, the function $f: \mathbb{R} \rightarrow \mathbb{R}$ defined by

$$
f(\eta)=(1+\theta) \eta+\int_{f(\eta)}^{\infty} \frac{1+\theta-y(s)}{y(s)} d s
$$

is the required similarity solution.

Note that the monotonicity of $y$ on $\mathbb{R}$ implies that

$$
f(\eta)>(1+\theta) \eta \quad \text { and } \quad f^{\prime \prime}(\eta)>0 \text { for all } \eta \in \mathbb{R} \text {. }
$$

To obtain a second lower bound for $f$ we write (5.7) as

$$
\begin{aligned}
f(\eta) & =(1+\theta) \eta+(1+\theta) A+\int_{f(\eta)}^{0} \frac{1+\theta-y(s)}{y(s)} d s \\
& =(1+\theta) \eta+(1+\theta) A+\int_{f(\eta)}^{0} \frac{d s}{y(s)}+\int_{f(\eta)}^{0} \frac{\theta-y(s)}{y(s)} d s \\
& =\theta \eta+\theta A-\int_{s(\eta)}^{0} \frac{y(s)-\theta}{y(s)} d s
\end{aligned}
$$


by (5.4) and (5.6). With

$$
B=\frac{1}{\theta} \int_{-\infty}^{0} \frac{y(s)-\theta}{y(s)} d s
$$

we can write $(5.8)$ as

$$
f(\eta)=\theta(\eta+A-B)+\int_{-\infty}^{f(\eta)} \frac{y(s)-\theta}{y(s)} d s .
$$

Remembering that $y(f)$ is strictly increasing we conclude that

$$
f(\eta)>\theta(\eta+A-B) \text { for all } \eta \in \mathbb{R} \text {. }
$$

For the asymptotic behaviour as $\eta \rightarrow-\infty$ we deduce from (5.10) that

$$
f(\eta)=\theta(\eta+A-B)+O\left(\eta^{-2} e^{-\gamma-\eta^{2}}\right) \text { as } \eta \rightarrow-\infty
$$

and that

$$
\begin{aligned}
\eta f^{\prime}(\eta)-f(\eta) & =\eta \nu(f)-f \\
& =-A+B+O\left(e^{-\gamma-f^{2}}\right) \text { as } f \rightarrow-\infty
\end{aligned}
$$

The monotonicity of $y(f)$ implies that we can estimate $A$ and $B$ by

$$
\begin{gathered}
A<\frac{1}{\theta(1+\theta)} \int_{0}^{\infty}\{1+\theta-y(s)\} d s, \\
B>\frac{1}{\theta(1+\theta)} \int_{-\infty}^{0}\{y(s)-\theta\} d s .
\end{gathered}
$$

However, when we integrate (5.2) over $(-\infty, \infty)$ and use the fact that $y^{\prime}(f) \rightarrow 0$ as $f \rightarrow \pm \infty$, we find that

$$
\int_{-\infty}^{\infty} f y^{\prime}(f) d f=0
$$

or

$$
\int_{\theta}^{1+\theta} \tau(y) d y=0
$$

where, as in section $3, \tau$ is the inverse of $y$. This means that

$$
\int_{-\infty}^{0}\{y(f)-\theta\} d f=\int_{0}^{\infty}\{1+\theta-y(f)\} d f
$$

and so, in view of (5.11) and (5.12), that

$$
B-A>0 \text { for all } \lambda \geqslant 0 \text { and } \theta>0 .
$$


From the asymptotic behaviour for $\eta \rightarrow \infty$ one finds that

$$
\lim _{z \rightarrow-\infty} v(x, z)=\frac{B-A}{2 \sqrt{ } x} \text { for all } x>0 .
$$

Thus (5.13) tells us that in this case, salt water is also drawn into the mixing zone.

Remarks. (i) The stream function $\psi$ vanishes when $f$ vanishes. Thus, let $f(\hat{\eta})=0$. Then we deduce from (5.7) that

$$
\hat{\eta}=-\frac{A}{1+\theta}
$$

This implies that

$$
\psi(x, z)>(<) 0 \text { when } z>(<)-\frac{A}{1+\theta} \sqrt{ } x .
$$

(ii) Because we imposed the condition $v(x, z) \rightarrow 0$ as $z \rightarrow \infty$ we find for $\theta \geqslant 0$ that salt water is drawn into the mixing zone. We might equally well have started out by assuming that $v(x, z) \rightarrow 0$ as $z \rightarrow-\infty$. The fresh water would then have been drawn into the mixing zone.

\section{REFERENCES}

1. J. Bear, Dynamics of Fluids in Porous Media (Elsevier, New York 1972).

2. - Hydrodynamics of Ground Water (McGraw-Hill, New York 1979).

3. G. DE Josseling De JoNG and C. J. van Duij, J. Hydrology 84 (1986) 55-79.

4. G. J. M. Uffink, In Proceedings, Ninth Salt Water Intrusion Meeting (ed. R. H. Boekelman et al:; Delft University of Technology, Delft 1986).

5. -..., Analysis of dispersion by the random-walk method. Ph.D. Thesis, Delft 1990.

6. G. DE Jossling DE JonG, In Proceedings, Euromech 143 (ed. A. Verruijt and F. B. J Barends; Balkema, Rotterdam 1981).

7. R. voN Mises and K. O. Friedrichs, Fluid Dynamics, Applied Mathematical Sciences 5 (Springer, New York 1971).

8. H. Schlichting, Boundary Layer Theory, 6th edition (McGraw-Hill, New York 1968).

9. R. Iglisch, Z. angew. Math. Mech. 33 (1953) 143-147.

10. D. G. Aronson, The Porous Medium Equation, Some Problems in Nonlinear Diffusion, Lecture Notes in Mathematics (ed. A. Fasano and M. Primicerio; Springer, Berlin 1987).

11. L. A. Peletier, Arch. ration. Mech. Analysis 56 (1974) 183-190.

12. F. V. Atkinson and L. A. Peletier, ibid. 42 (1972) 369-379.

13. - - and — ibid. 54 (1974) 373-392.

14. C. J. van Duijn, S. M. Gomes and Zhang Hongfei, IM A J. appl. Math. 41 (1988) $147-163$.

15. — and L. A. Peletier, J. nonlinear Anal. TMA 1 (1977) 223-233. 